\title{
Chest pain in a 12-year-old boy: when is it a harbinger of poor outcome?
}

\author{
Hisham Alomran • Faisal AlGhamdi • \\ Fadiah AlKhattabi
}

Received: 22 January 2009 / Accepted: 16 April 2009 /Published online: 3 June 2009

(C) Springer-Verlag London Ltd 2009

\begin{abstract}
Chest pain is usually a benign presentation in children who present to emergency departments (ED) or primary care centers. Unlike adults, where chest pain is commonly due to cardiac causes, in children the cause is more likely secondary to non-cardiac causes. Here we present a case of a child known to have hyper-eosinophilic syndrome (HES) who presented with sudden onset of chest pain and had a rapidly progressive and fatal outcome in the ED. We discuss the ED approach to the child with chest pain and review acute myocardial infarction (AMI) in children.
\end{abstract}

\section{Case history and course}

A 12-year-old boy known to have idiopathic HES presented to our ED with a complaint of cough, shortness of breath (SOB), and severe chest tightness for the previous $1 \mathrm{~h}$. He had been diagnosed with refractory HES 4 years back, with a course complicated by multiple organ involvement. Previous medications included cyclosporine, prednisolone, hydroxyurea, and imatinib. At the time of his current presentation he was on prednisolone $10 \mathrm{mg}$ t.i.d., cyclosporin A $50 \mathrm{mg}$ b.i.d., hydroxyurea $750 \mathrm{mg}$ o.d., and ciprofloxacillin $250 \mathrm{mg}$ o.d.

H. Alomran $(\bowtie) \cdot$ F. AlKhattabi

King Faisal Specialist Hospital,

Riyadh, Saudi Arabia

e-mail: hisham_omran@hotmail.com

F. AlGhamdi

King Fahad Medical City,

Riyadh, Saudi Arabia
The chest tightness was acute and later became more consistently associated with the chest pain. The pain was localized to the central right side and although not quantified was severe enough to make him cry. It was non-radiating in character and aggravated by breathing. The cough was described as productive of yellowish phlegm without blood. He denied fever or sweating.

His vital signs were as follows: heart rate $124 \mathrm{bpm}$, blood pressure 108/92 $\mathrm{mmHg}$, respiratory rate 32 breaths/min, temperature $36.3^{\circ} \mathrm{C}$. In general he appeared anxious and in significant pain, grasping his chest. Pulses were regular and prominent. He had good and equal air entry, with bilateral crackles. There was no wheezing. The heart sounds were regular S1 and S2 without any additional sounds. The chest wall was non-tender. The abdomen was non-tender and without any organomegaly. The patient was oriented and coherent; no focal deficits on cranial nerve exam. He was noted to have a chronic extensive eczematous rash involving the trunk and abdomen.

Our patient received two doses of albuterol nebulizer treatments. As the severity of the chest pain progressed, treatment with $\mathrm{O}_{2}$ via nasal cannula was initiated and intravenous access was established.

The first ECG, done $20 \mathrm{~min}$ after his presentation, is shown in Fig. 1. At that time the presumptive diagnosis of AMI was entertained. Morphine $2 \mathrm{mg}$ intravenously was initiated as well as aspirin $100 \mathrm{mg}$ po. Nitroglycerin and heparin were also ordered but were not administered. His labs showed: WBC $22.5 \times 10^{9} / 1$ and neutrophils $59 \%$, hemoglobin $77 \mathrm{~g} / 1$ (normal: 110-149 g/l), pH 7.21 (normal: 7.35-7.45), $\mathrm{HCO}_{3}^{-} 5.9 \mathrm{mmol} / \mathrm{l}$, urea $4.4 \mu \mathrm{mol} / \mathrm{l}$ (normal: < $8 \mu \mathrm{mol} / \mathrm{l}$ ), creatinine $59 \mu \mathrm{mol} / \mathrm{l}$ (normal: $53-88 \mu \mathrm{mol} / \mathrm{l}$ ), creatinine kinase 230 units/l (normal: 40-170 units/l), troponin $2 \mu \mathrm{g} / \mathrm{l}$ (normal range: $<0.35 \mu \mathrm{g} / \mathrm{l}$ ). Other investigations were within normal limits. 


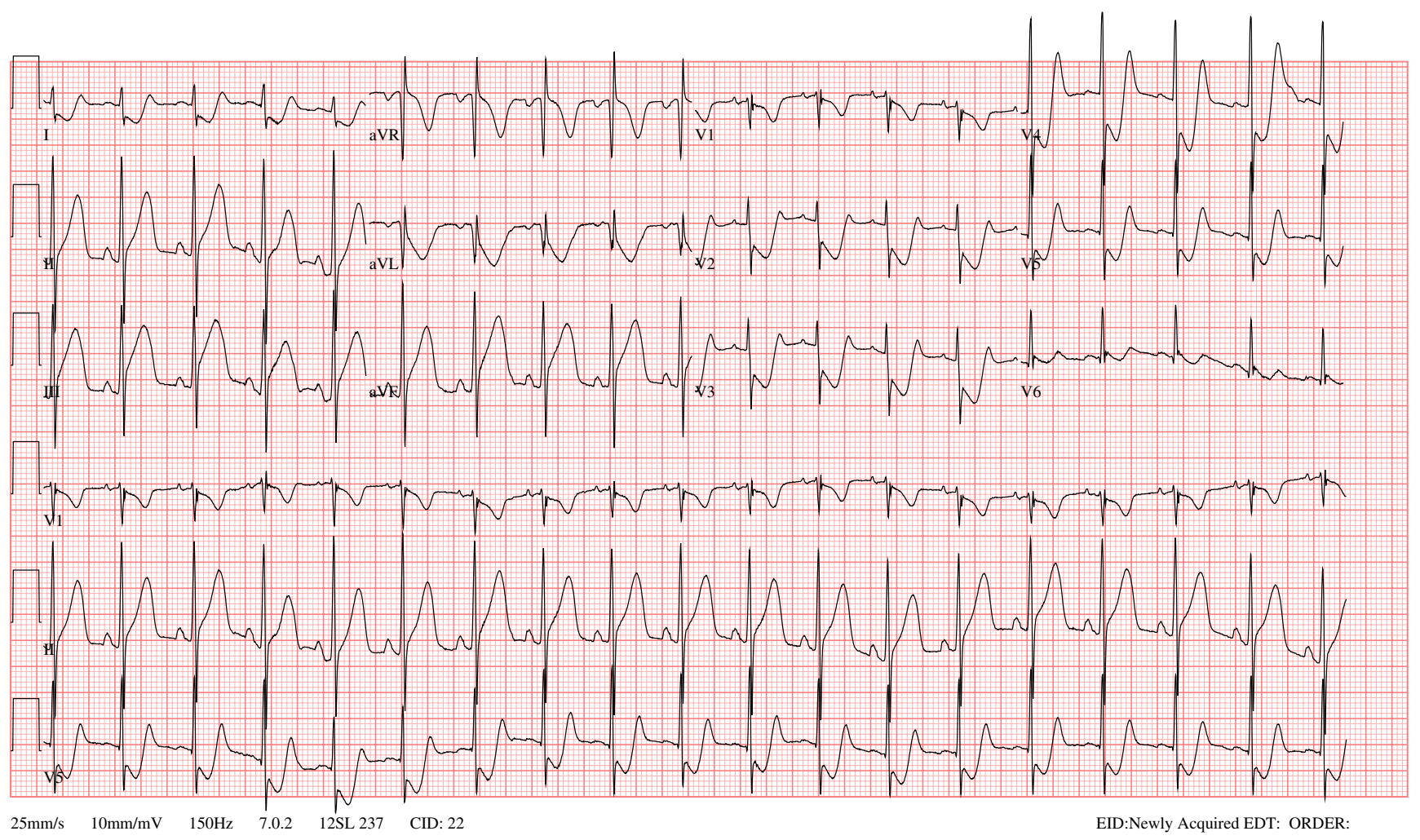

Fig. 1 First ECG done 20 min after the patient's presentation

Approximately 20 min later, the child's ECG showed the patterns in Figs. 2 and 3. The patient's condition deteriorated with the development of hypotension, weak pulses, and a decrease in level of consciousness.

Resuscitation included intubation and the activation of the advanced cardiac resuscitation protocol. He received three doses of atropine $0.5 \mathrm{mg}$, ketamine $2 \mathrm{mg}$ and rocuronium $25 \mathrm{mg}$ and four doses of epinephrine $(0.3 \mathrm{mg}$ each). His condition continued to progress without response to therapy and the patient died after developing a terminal ventricular rhythm.

\section{Discussion}

Despite the rarity of serious pathological conditions in children presenting with chest pain, the rapidity of progression of our patient's condition illustrates the need to develop a systematic approach to the child who presents with a complaint of chest pain in the ED.

ED incidence of chest pain in children is much less common $(0.06 \%)$ as compared to adults (2.21\%) [1-3] and occurs equally in boys and girls with a mean age of 12 years. Studies have shown that, unlike adults, the cause of chest pain in children is rarely due to cardiac causes [4], but as illustrated by our case the complaint is not always benign, and serious cardiac and noncardiac causes must be identified.
Causes of chest pain in children are diverse and the role of the ED physician is to differentiate between the minor causes and those that pose a threat to life, and to act in a timely manner to stabilize the patient [5]. A suggested way to classify this symptom is to consider cardiac and noncardiac causes of chest pain (Tables 1 and 2). These tables show the diverse causes of chest pain in children, and as such only a thorough history and physical examination with an understanding of the various etiologies will lead to the correct diagnosis of the cause of the chest pain. In a small percentage of children, laboratory or radiologic or other invasive interventions will be required to better define the cause of the symptoms.

It is important to note at this time that the history and physical examination of a child with chest pain can be particularly problematic. Firstly, parental anxiety commonly is associated with the child with chest pain. This may be due to parental concern that the child is developing an ischemic event similar to that associated with atherosclerosis in adults. Secondly, children may not be able to describe the effectively (eg. quality of pain, associated symptoms). Also, the physical examination, as always in children, can be hampered by an anxious or crying child. Therefore, the examining physician needs to make a special effort to comfort both the child and the parents to maximize the chances of a thorough history and physical examination. 


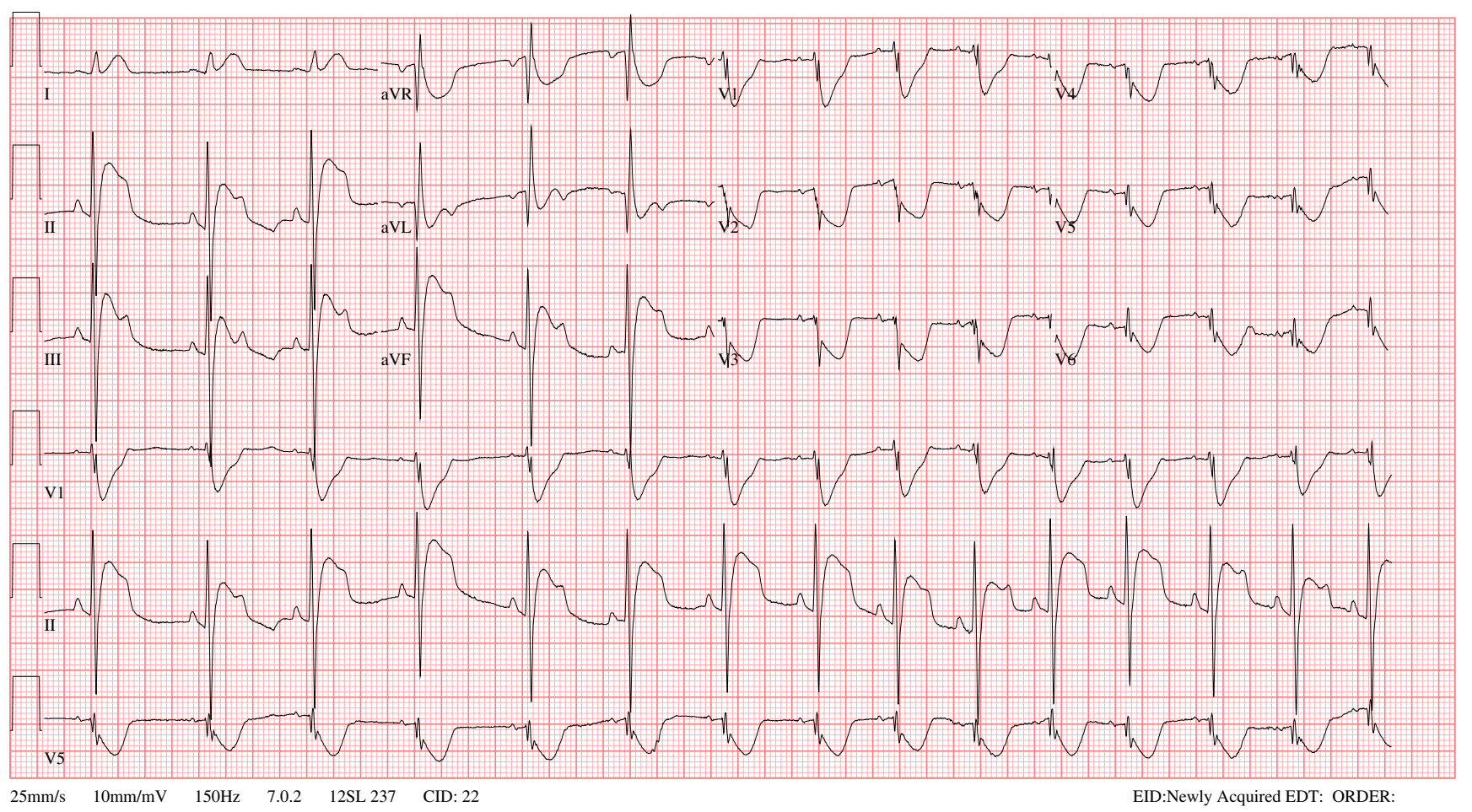

Fig. 2 ECG approximately 10 minutes after initial ECG

A detailed history of the chest pain should include the character of the pain, location, radiation, duration, and frequency as well as activities that exacerbate or alleviate the pain.

Generally, musculoskeletal chest pain is sharp and well localized and is commonly exacerbated by positional changes or by inspiration. In the adult literature, the reproducibility of the pain by palpation of the chest wall has been found to not be specific enough to rule out coronary artery disease [6-8], although in children such data have not been validated. We find that chest pain in children is commonly related to vague or distant chest trauma or carrying of heavy objects such as school bags.

Cardiac chest pain is generally described as crushing, squeezing, or pressure-like and is usually exacerbated by exertion. It may radiate to the shoulder, arm, neck, or jaw.

Relief of pain when sitting up may indicate pericardial causes of chest pain while worsening of pain with movement or deep inspiration is more indicative of noncardiac causes. The presence of cough, fever, or dyspnea may suggest pulmonary causes of the pain while worsening of the pain post meals, or when lying down, may suggest gastroesophageal reflux disease.

Additional historical factors useful in evaluating a child with chest pain include symptoms such as shortness of breath, diaphoresis, and nausea. These symptoms are commonly associated with cardiac causes of chest pain, but are not specific or sensitive independently.
Syncope and palpitations are additional symptoms which may be difficult to elicit in children but if present may be indicative of cardiac causes such as dysrhythmia, ischemia, or outflow tract obstruction.

Other historical facts of importance include the presence of hypertension, diabetes, obesity, asthma, previous thromboembolic phenomena, hypercholesterolemia, gastroesophageal reflux, Marfan syndrome, and Kawasaki disease.

Patients with recent cardiac surgery and those with congenital cardiac disease should be assessed carefully for cardiac causes of chest pain which may include pericardial effusion, dysrhythmias, and ischemia. Patients with metabolic syndromes or family history of syndromes such as lipoprotein deficiencies and homocystinuria are also predisposed to have ischemia and therefore should be assessed thoroughly with further investigations.

A complete list of current and past medications has to be obtained, and in particular the use of corticosteroids or other immunosuppressants which have been associated with the development of ischemic heart disease, as well as the previous or current use of oral contraceptives which may predispose the child to atherosclerosis. A family history of hypercoagulable states such as proteins $\mathrm{S}$ and $\mathrm{C}$ deficiency or antiphospholipid syndromes are important pieces of the history which predispose to thromboembolic phenomena such as pulmonary embolism and ischemic heart disease.

Finally, in adolescents with chest pain, it is vital to ask about substance abuse (crack, cocaine, or amphetamines) as well as the sniffing of solvents which have all been 


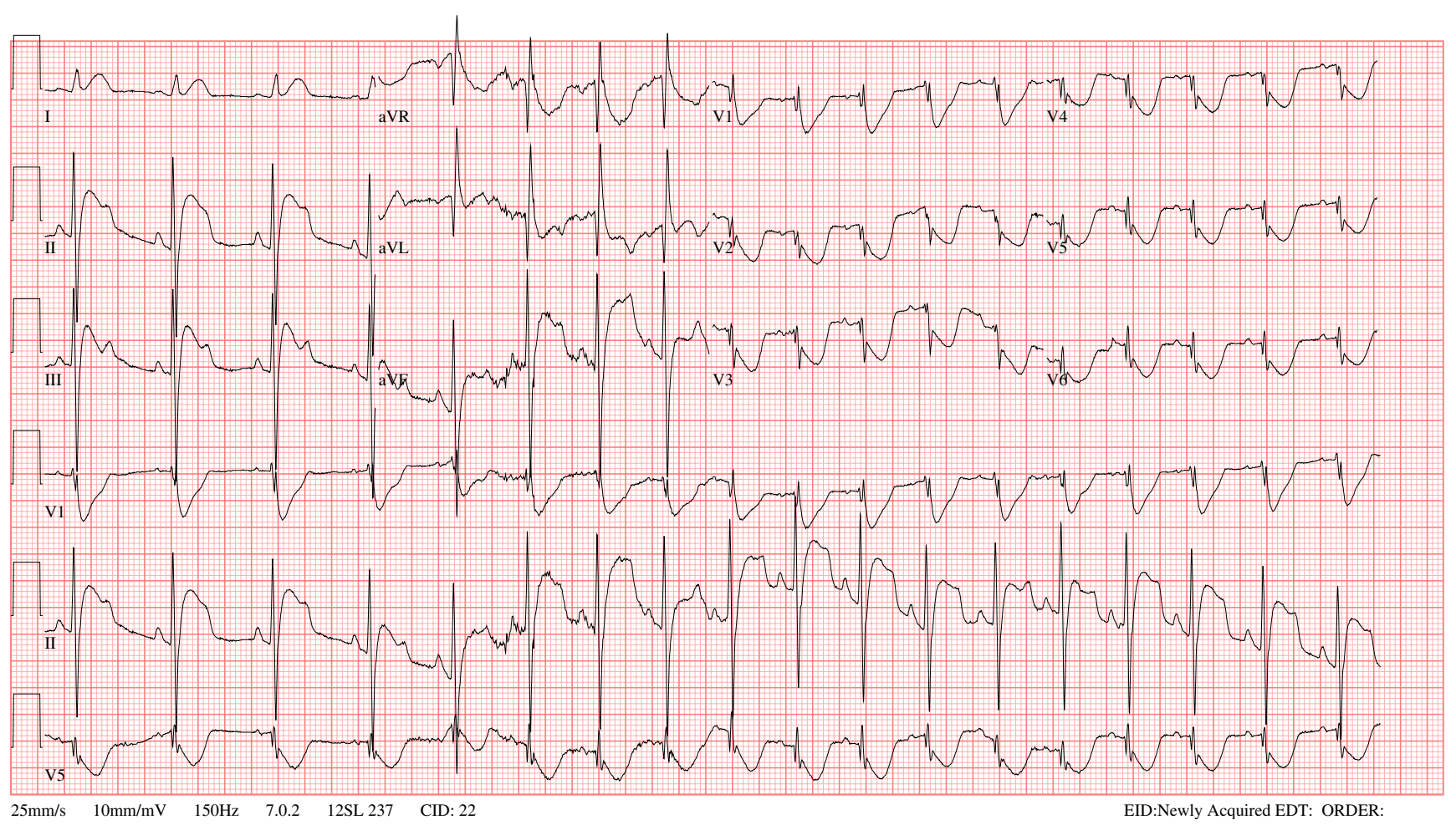

Fig. 3 ECG approximately $20 \mathrm{~min}$ after the initial ECG

associated with development of ischemic heart disease and chest pain.

The general examination should identify the child in severe distress who needs immediate treatment for lifethreatening conditions. This starts with evaluation of the airway, breathing, and circulation (ABCs) as well as the presenting vital signs. For example, a gasping or cyanotic patient needs immediate intervention prior to completion of the physical examination, which may consist of simply giving oxygen or establishing an airway to relieve obstruction. Once this is stabilized, the patient's vital signs need to be reviewed carefully. The importance of the vital signs cannot be overstated and a good rule is any abnormality of the vital signs should be investigated or the cause clarified prior to discharge of a patient.

Although the respiratory rate is a very sensitive tool for identifying patients with pneumonia, an increased respiratory rate (in accordance to the norms for age) was found to have a sensitivity of $71-83 \%$ in different age groups and a negative predictive value of $96-99 \%$ in pneumonia $[9,10]$, but was not specific enough to rule out other etiologies.

The temperature may be useful in identifying children with infectious or autoimmune causes of chest pain, such as pneumonia, pleural effusions, pericarditis, or myocarditis.

The presence of tachycardia may be due to a variety of causes and does not serve to differentiate between cardiac and noncardiac causes of chest pain. Although a signifi- cantly high heart rate for age (generally above $200 \mathrm{bpm}$ in children) should raise the concern for dysrhythmias.

Pulse oximetry commonly known as the fifth vital sign is a particularly helpful tool and abnormalities have to be taken seriously. Although not specific, serious causes include pneumonia, pneumonitis, and pulmonary embolism as well as nonspecific cardiac dysfunction

A complete chest and cardiovascular examination is essential. The chest wall should be evaluated for signs of trauma, tenderness (suggesting musculoskeletal pain), or subcutaneous air (suggesting a pneumothorax or pneumomediastinum). There may be rales, wheezes, or decreased breath sounds if there is a pulmonary pathological condition or murmurs, rubs or muffled heart sounds if there is a cardiac pathological condition.

Based on the findings on the history and physical examination, the physician should decide whether the patient requires further investigation. These could include a chest X-ray (anteroposterior and lateral), laboratory investigations, and/or an ECG. The chest X-ray will help confirm the presence of rib fractures, pneumonia, pneumothorax, pleural and pericardial effusions, while the laboratory investigations may suggest infectious processes. Patients with a higher suspicion of cardiac etiology of chest pain may require measurement of creatine kinase isoenzyme muscle-brain (CK-MB) fraction and/or troponins. Troponin is a particularly sensitive and specific marker of myocardial ischemia as it is only released from 
Table 1 Noncardiac causes of pediatric chest pain

Musculoskeletal disorders
- Chest wall strain
- Direct trauma/contusion
- Rib fracture
- Costochondritis
- Slipping rib syndrome
Respiratory disorders
- Severe cough
- Asthma
- Pneumonia
- Pneumothorax/pneumomediastinum
- Pulmonary embolism
Psychologic disorders
- Stress-related pain
Gastrointestinal disorders
- Reflux esophagitis
- Esophageal foreign body
Miscellaneous disorders
- Sickle cell crises
- Abdominal aortic aneurysm (Marfan syndrome)
- Pleural effusion (collagen vascular disease)
- Shingles
- Pleurodynia (coxsackievirus)
- Breast tenderness (pregnancy, physiologic)
Idiopathic

damaged myocardium unlike $\mathrm{CK}$ and lactic dehydrogenase (LDH). In our patient, the troponin level was $2 \mu \mathrm{g} / \mathrm{l}$. The normal range is $<0.35 \mu \mathrm{g} / \mathrm{l}$.

The ECG is a sensitive tool to identify many cardiac causes such as dysrhythmia and ischemia as well as pulmonary causes such as pulmonary embolism which can present with S1 Q3 and inverted T3. These findings are specific but not sensitive and it is important to note that the normal ECG does not rule out a cardiac pathological condition.

Our patient's ECG showed ST depression, in the anterolateral leads V2-V6 (Fig. 1). These findings are classically consistent with anterolateral AMI. This pattern progressed in Fig. 2 with further evidence of expansion of the myocardial ischemia.

In contrast to the ECG in adults, where electrocardiographic criteria for the diagnosis of cardiac ischemia is well defined, analogous criteria in infants and children are ambiguous. The difficulty relates to age-dependent differences in the pediatric electrocardiogram, the presence or absence of congenital heart disease, and multiple and diverse etiologies of myocardial injury that lead to an ischemic pattern on the ECG [11].

Other invasive diagnostic studies such as cardiac catheterization or cardiac biopsy will be dictated by the condition of the patient and the decision of the cardiologist. These procedures may be useful in confirming causes of AMI, whether it is due to coronary ischemia or myocarditis [12], and in addition, catheterization provides the opportunity of opportunity of revascularization therapy.

AMI in children is rare and the causes vary depending on the age at presentation (Table 3). Overall, the most common cause of AMI in children is anomalous origin of the left coronary artery, which may present in the neonatal period with unexplained sudden death, or later with persistent irritability, or evidence of heart failure. Myocarditis, the most likely cause of our patient's presentation, is a frequently missed cause of myocardial ischemia with an incidence of only $0.012 \%$ of patients seen in the ED but with a postmortem examination prevalence of $17 \%$ in children with sudden or unexpected deaths. [13, 14].

Although thrombotic diseases present similarly with AMI and/or heart failure, the age at incidence of thrombotic coronary disease depends on the primary disease. For example, thrombotic disease due to Kawasaki disease will occur earlier than thrombosis due to hypercoagulable states which usually peak at puberty. Other rarer causes are related to postoperative complications of congenital heart disease such as following repair of D-transposition of the great arteries (TGA).

Although the causes of myocardial ischemia may be different than in adults, the management is similar. This

Table 2 Cardiac causes of pediatric chest pain

Coronary artery disease-ischemia/infarction

- Anomalous coronary arteries

- Coronary arteritis (Kawasaki disease)

- Ischemic heart disease

Arrhythmia

- Supraventricular tachycardia

- Ventricular tachycardia

Structural abnormalities

- Hypertrophic cardiomyopathy

- Severe pulmonic stenosis

- Aortic valve stenosis

- Mitral valve prolapse

Infection

- Pericarditis

- Myocarditis 
includes starting oxygen therapy, establishing intravenous access, and close monitoring. Specific therapy includes relief of pain and anxiety and decreasing catecholamine response. Aspirin or other antiplatelet agents should be given on arrival. Morphine sulfate and nitroglycerin (sublingual, spray, or intravenous) are the first-line antianginal medications. These should be titrated until the symptoms subside or side effects develop [15].

Depending on the patient's condition, heart failure or antiarrhythmic agents may need to be added. We found no data supporting the use of beta blockers in children with AMI.

A mainstay of therapy in adults with evidence of acute myocardial ischemia is reperfusion therapy (medical) or definitive percutaneous cardiac interventions (PCI), depending on the patient's condition, access to cardiac catheter labs, other support services, and expertise of the treating cardiologists. The performance of cardiac catheterization may have provided the opportunity for revascularization therapy, but this was not pursued because of the limited expertise in performing this diagnostic and therapeutic procedure in the unstable patient at our institute. Although our patient died rapidly, the question of whether an adult with a similar presentation would have been managed similarly is an important one. Importantly, the cause for such a presentation in an adult is almost always coronary in origin. Therefore, the opportunity for diagnosis and therapy with PCI or thrombolysis is much more clear as compared to the child who may have evidence of myocardial injury but with presumably healthy coronary arteries.

Our case is interesting because of the rarity of such a rapid tragic outcome in a child with a seemingly benign complaint of chest pain. This illustrates the importance of always considering the broad range of causes of chest pain in children even when the majority of the patients are likely to have less dramatic sequelae. In addition, the presence of underlying disease should always increase awareness of the potential of multisystem involvement.
Patient was known to have a long-standing HES, which refers to a group of leukoproliferative disorders characterized by an overproduction of eosinophils that results in organ damage of virtually any organ $[16,17]$. Our patient had previous involvement of his pulmonary and integumentary system but never the cardiovascular system. The development of cardiac disease in children with HES is not predictable, and the severity of cardiac injury does not clearly correlate with the degree of peripheral eosinophilia [18]. Patients with cardiac involvement due to HES may present with symptoms of dyspnea, chest pain, signs of heart failure, mitral or tricuspid regurgitation, or cardiomegaly. Although all layers of the heart can be affected in HES, it is believed the myocardial involvement is the most common.

On rare occasions, as in our patient, patients present with evidence of acute AMI. It is this infarction, as evidenced by the ECG changes and the rise in troponin, which was the suspected cause of the chest pain and the devastating outcome. In AMI, the ECG findings could be subtle such as $\mathrm{T}$-wave inversion or with more classic evidence of ischemia. Echocardiography may demonstrate intracardiac thrombi and evidence of fibrosis and increase in endomyocardial echo density in areas of fibrosis in addition to evidence of acute ischemia. Although the exact cause of the myocardial ischemia could not be confirmed without either cardiac catheterization or obtaining a tissue biopsy, it is suspected this AMI pattern was due to acute myocarditis.

In HES, the underlying cardiac pathological condition is thought to be a myocarditis secondary to eosinophilic infiltration of the myocardium (eosinophilic myocarditis, EM). EM evolves through three stages: an acute necrotic stage, an intermediate phase characterized by thrombus formation along the damaged endocardium, and the final fibrotic stage. Due to the acuity of our patient's presentation, we suspect our patient was in the acute necrotic stage, but no tissue diagnosis was obtained. The possibility of toxin-related myocarditis (due to previous and current use of immunosuppressants) or coronary artery obstruction cannot be ruled out.
Table 3 Causes of acute myocardial infarction in children

\begin{tabular}{ll} 
Causes & \\
\hline Anomalous left coronary artery & Takayasu's disease \\
Coronary artery stenosis or spasm & Cardiomyopathy \\
Myocarditis & Marfan syndrome \\
Cardiac trauma & Kawasaki disease \\
Sickle cell disease & Illicit drugs, cocaine, amphetamine \\
Nephritic syndrome & Homocystinuria \\
Repair of D-TGA & Tetralogy of Fallot \\
Toxin & Autoimmune
\end{tabular}


In general, immunosuppressants have a wide range of cardiacrelated complications such as myocarditis, and corticosteroids in particular, which are the most commonly used immunosuppressants, have been associated with a wide range of cardiac complications such as cardiomyopathy or coronary thrombosis due to hypercoagulable states.

\section{Conclusion}

From this case, we conclude that physicians dealing with the pediatric population will commonly deal with patients who present with chest pain, the cause of which may be life threatening. The approach to such a scenario requires a thorough history and physical exam and a risk assessment of the patient. If the risk is deemed to be significant, then more investigations and consultations may be warranted.

Although not done in our patient, when presented with biochemical evidence of myocardial injury and an ECG consistent with myocardial infarction in a child, more invasive procedures such as cardiac catheterization, endomyocardial biopsy, or autopsy should be performed to differentiate between the various causes of myocardial tissue damage. The timing and location of such interventions will be dictated by the medical condition of the patient and the expertise of the supporting staff. Treatment should be started as early as possible and may require consultation with those with more experience managing myocardial injury.

\section{References}

1. Selbst SM, Ruddy RM, Clark BJ et al (1988) Pediatric chest pain: a prospective study. Pediatrics 82:319-323

2. Leung AKC, Giuffre RM (2004) Pediatric chest pain. Clin Pediatr (Phila) 43(9):863

3. Selbst SM (1997) Consultation with the specialist. Chest pain in children. Pediatr Rev 18:169-173

4. Reich JD, Campbell R (1998) Myocardial infarction in children. Am J Emerg Med 16:296-303

5. Lee TH, Goldman L (2000) Evaluation of the patient with acute chest pain. N Engl J Med 342:1187-1195
6. Pope JH, Ruthazer R, Beshansky JR et al (1998) Clinical features of emergency department patients presenting with symptoms suggestive of acute cardiac ischemia: a multicenter study. J Thromb Thrombolysis 6:63-74

7. Swap CJ, Nagurney JT (2005) Value and limitations of chest pain history in the evaluation of patients with suspected acute coronary syndromes. JAMA 294:2623-2630

8. Goodacre S, Locker T, Morris F, Campbell S (2002) How useful are clinical features in the diagnosis of acute, undifferentiated chest pain? Acad Emerg Med 9:203-208

9. Taylor JA, Del Beccaro M, Done S, Winters W (1995) Establishing clinically relevant standards for tachypnea in febrile children younger than 2 years. Arch Pediatr Adolesc Med 149:283-287

10. Rothrock SG, Green SM, Fanelli JM, Cruzen E, Costanzo KA, Pagane J (2001) Do published guidelines predict pneumonia in children presenting to an urban ED? Pediatr Emerg Care 17 (4):240-243

11. Gazit AZ, Avari JN, Balzer DT, Rhee EK (2007) Electrocardiographic diagnosis of myocardial ischemia in children: is a diagnostic electrocardiogram always diagnostic? Pediatrics 120:440-444

12. Sirois JG (1995) Acute myocardial infarction. Emerg Med Clin North Am 13:759-769

13. Hoyer MH, Fischer DR (1991) Acute myocarditis simulating myocardial infarction in a child. Pediatrics 87:250-253

14. Galiuto L, Enriquez-Sarano M, Reeder GS, Tazelaar HD et al (1997) Eosinophilic myocarditis manifesting as myocardial infarction: early diagnosis and successful treatment. Mayo Clin Proc 72:603-610

15. Simoons ML, Boersma E, Maas AC et al (1997) Management of myocardial infarction: the proper priorities. Eur Heart J 18:896899

16. Weller PF, Bubley GJ (1994) The idiopathic hypereosinophilic syndrome. Blood 83:2759-2779

17. Chusid MJ, Dale DC, West BC, Wolff SM (1975) The hypereosinophilic syndrome: analysis of fourteen cases with review of the literature. Medicine (Baltimore) 54:1-27

18. Take M, Sekiguchi M, Hiroe M et al (1985) Clinical spectrum and endomyocardial biopsy findings in eosinophilic heart disease. Heart Vessels Suppl 1:243-249

Faisal AlGhamdi is an Associate Consultant in the Pediatric Emergency Department at King Fahad Medical City, Riyadh, Saudi Arabia. He is board certified in Pediatric Emergency Medicine from a local training program in Saudi Arabia and currently is a fellow at the Hospital for Sick Children in Toronto, Canada. He has an interest in medical education and is planning to earn a master's degree in that field. 